\title{
CReasPy-Cloning: A Method for Simultaneous Cloning and Engineering of Megabase-Sized Genomes in Yeast Using the CRISPR-Cas9 System
}

Estelle Ruiz, ${ }^{\dagger, \ddagger}$ Vincent Talenton, ${ }^{\dagger,}$ Marie-Pierre Dubrana, ${ }^{\dagger, \ddagger}$ Gabrielle Guesdon, ${ }^{\dagger, \ddagger}$

Maria Lluch-Senar, ${ }^{\S, \|}$ Franck Salin, ${ }^{\perp}$ Pascal Sirand-Pugnet, ${ }^{\dagger,}$ Yonathan Arfi, ${ }^{\dagger, \ddagger}$ and Carole Lartigue ${ }^{*}, \dagger, \ddagger \odot$

${ }^{\dagger}$ INRA, UMR 1332 de Biologie du Fruit et Pathologie, F-33140 Villenave d’Ornon, France

${ }^{\ddagger}$ Univ. Bordeaux, UMR 1332 de Biologie du Fruit et Pathologie, F-33140 Villenave d'Ornon, France

${ }^{\S}$ EMBL/CRG Systems Biology Research Unit, Centre for Genomic Regulation (CRG), The Barcelona Institute of Science and

Technology, Dr Aiguader 88, Barcelona 08003, Spain

"Universitat Pompeu Fabra (UPF), 08003 Barcelona, Spain

${ }^{\perp}$ BIOGECO, INRA, Univ. Bordeaux, 33610 Cestas, France

Supporting Information

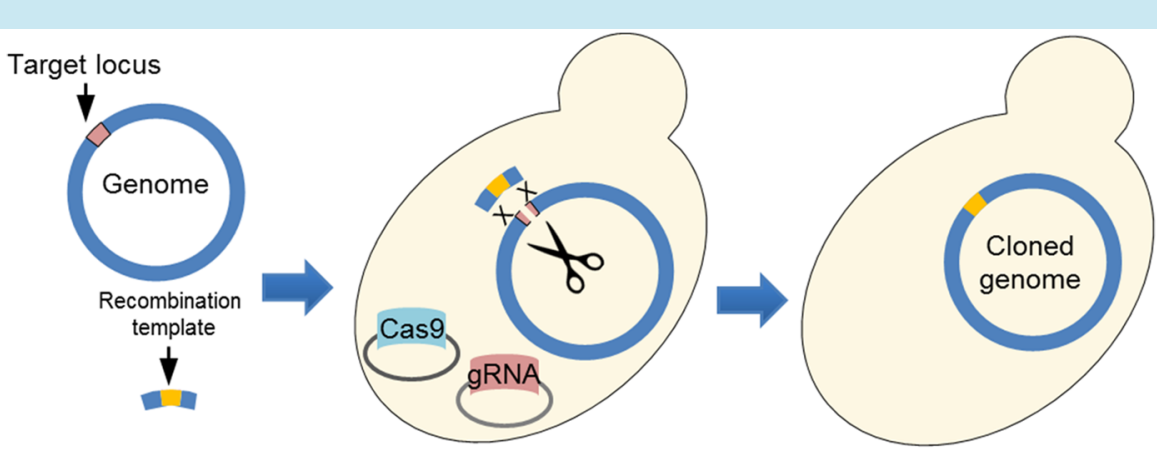

ABSTRACT: Over the past decade, a new strategy was developed to bypass the difficulties to genetically engineer some microbial species by transferring (or "cloning") their genome into another organism that is amenable to efficient genetic modifications and therefore acts as a living workbench. As such, the yeast Saccharomyces cerevisiae has been used to clone and engineer genomes from viruses, bacteria, and algae. The cloning step requires the insertion of yeast genetic elements in the genome of interest, in order to drive its replication and maintenance as an artificial chromosome in the host cell. Current methods used to introduce these genetic elements are still unsatisfactory, due either to their random nature (transposon) or the requirement for unique restriction sites at specific positions (TAR cloning). Here we describe the CReasPy-cloning, a new method that combines both the ability of Cas9 to cleave DNA at a user-specified locus and the yeast's highly efficient homologous recombination to simultaneously clone and engineer a bacterial chromosome in yeast. Using the $0.816 \mathrm{Mbp}$ genome of Mycoplasma pneumoniae as a proof of concept, we demonstrate that our method can be used to introduce the yeast genetic element at any location in the bacterial chromosome while simultaneously deleting various genes or group of genes. We also show that CReasPy-cloning can be used to edit up to three independent genomic loci at the same time with an efficiency high enough to warrant the screening of a small $(<50)$ number of clones, allowing for significantly shortened genome engineering cycle times.

KEYWORDS: genome cloning, genome editing, CRISPR-Cas9, genome transplantation, mycoplasma, Saccharomyces cerevisiae

G enetically engineering a living organism is a key technology, for both fundamental and applied purposes. The ability to specifically delete, replace, or add a sequence to a genome can be used to study the function of a given gene or group of genes, ${ }^{1}$ or to add new or improved functions to a cell. ${ }^{2}$

From early groundbreaking experiments on bacterial transformation, ${ }^{3}$ to the most recent advances in genomic engineering using targetable nucleases, ${ }^{4,5}$ a wide array of tools have been developed in order to introduce and maintain exogenous genetic material in an organism or to directly edit the existing genome.
However, while multiple strategies are often available for the majority of model organisms, many species of biological interest still lack efficient genetic engineering tools. ${ }^{6}$

Over the past decade, it was proposed that these difficulties could be bypassed by transferring a whole genome to edit into another cell where efficient tools are available. ${ }^{7}$ This approach

Received: May 22, 2019

Published: October 30, 2019 
A
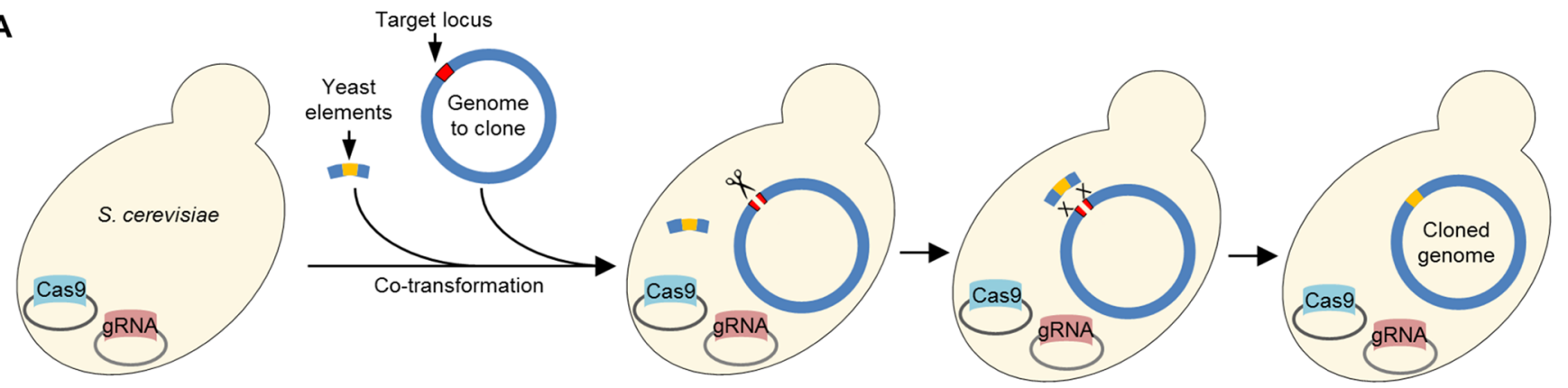

B
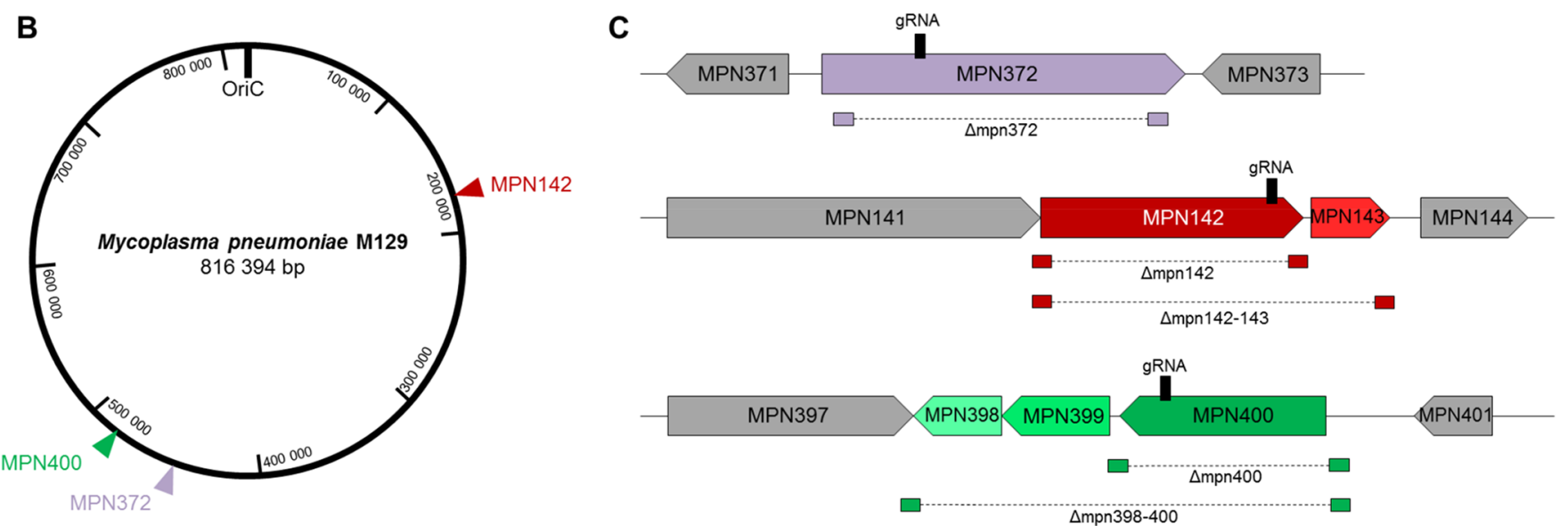

Figure 1. Overview of the CReasPy-cloning method and its application to M. pneumoniae M129 genome. (A) Schematic diagram of the experimental procedure of CReasPy-cloning. A yeast is transformed beforehand with two plasmids, allowing the expression of the Cas9 nuclease and a gRNA. PCas9 and pgRNA carry the TRP1 and URA3 selection marker, respectively. This yeast is then transformed simultaneously with the genome to be cloned and a linear fragment of DNA containing the yeast elements (CEN-HIS3, with or without ARS) flanked by two recombination arms homologous to each side of the target locus. Upon entry in the cell, the target genome is cleaved by the Cas $9 /$ gRNA complex, and subsequently repaired by the yeast homologous recombination system, using the provided linear DNA fragment as template. As a result, the bacterial genome now includes the yeast elements inserted at a precise locus, and is now carried by the yeast as an artificial chromosome. Yeast transformants are selected on the synthetic defined media SD-His. (B) Map of M. pneumoniae M129 genome. The location of the three targeted loci (MPN372, MPN142 and MPN400) is indicated by colored arrows. (C) Schematic view of the genomic regions surrounding the targeted loci. The location of the gRNA protospacer sequence is indicated by a black bar. The locations of the recombination arms are indicated by small rectangles connected by a dotted line.

was in particular developed using the yeast Saccharomyces cerevisiae as a genetic workbench to manipulate whole bacterial chromosomes. This process was also applied for the creation of the first bacterial cell governed by a chemically synthesized genome $^{8}$ and an engineered free-living organism with the smallest genome. ${ }^{9}$

Given the large array of genetic tools available in yeast, including TREC, ${ }^{10}$ TREC-IN, ${ }^{11}$ CRISPR-Cas9, ${ }^{12,13}$ this genome engineering strategy has generated a growing interest. To this date, a wide range of chromosomes from different organisms has been successfully cloned in yeast, originating from viruses, ${ }^{14,15}$ bacteria, ${ }^{16,17}$ and algae. ${ }^{18}$

In order to clone a bacterial chromosome in yeast, several elements must be added to it. These so-called "yeast elements" are a yeast centromere and a selection marker. In addition, one or several yeast origins of replication, also known as ARS (autonomously replicating sequence), must be added for genomes with high $\mathrm{G}+\mathrm{C} \%(>40 \%)$. For genomes with low GC content $(<40 \%)$, these ARS can be omitted as their AT rich consensus motif is naturally occurring in the genome. ${ }^{19-21}$ These yeast elements are required to drive the correct replication and maintenance of the foreign DNA, and are often provided as a single cassette that can be inserted into the bacterial genome using two main strategies. The first one relies on an initial transformation of the bacteria with a transposon bearing the yeast elements. ${ }^{7}$ While selection of living transformants ensures that the random integration of the transposon has not occurred in any locus essential for in vitro growth, this method cannot be used to integrate the yeast elements at a specific position of the genome. The second strategy is based on the transformation-associated recombination (TAR) cloning technique. $^{22}$ The complete bacterial genome is isolated in agarose plugs, linearized by restriction, and cotransformed into yeast together with the yeast element cassette flanked by recombination arms corresponding to both sides of the restriction locus. The yeast homologous recombination mechanism then circularize the bacterial chromosome by integration of the cassette. This approach necessitates the presence of a unique restriction site in the bacterial genome, preferably in a nonessential locus, which can be hard to find and limits the number of integration sites available.

In this study, we have developed a new strategy called CReasPy-Cloning, in order to efficiently insert the yeast elements at any desired locus of a DNA fragment to be cloned in yeast. This method expands on the logic of TAR cloning, by using the CRISPR/Cas9 system to generate a double strand break at a precise site of the DNA to clone. In addition, the flexibility offered by the choice of the insertion locus allows us to 

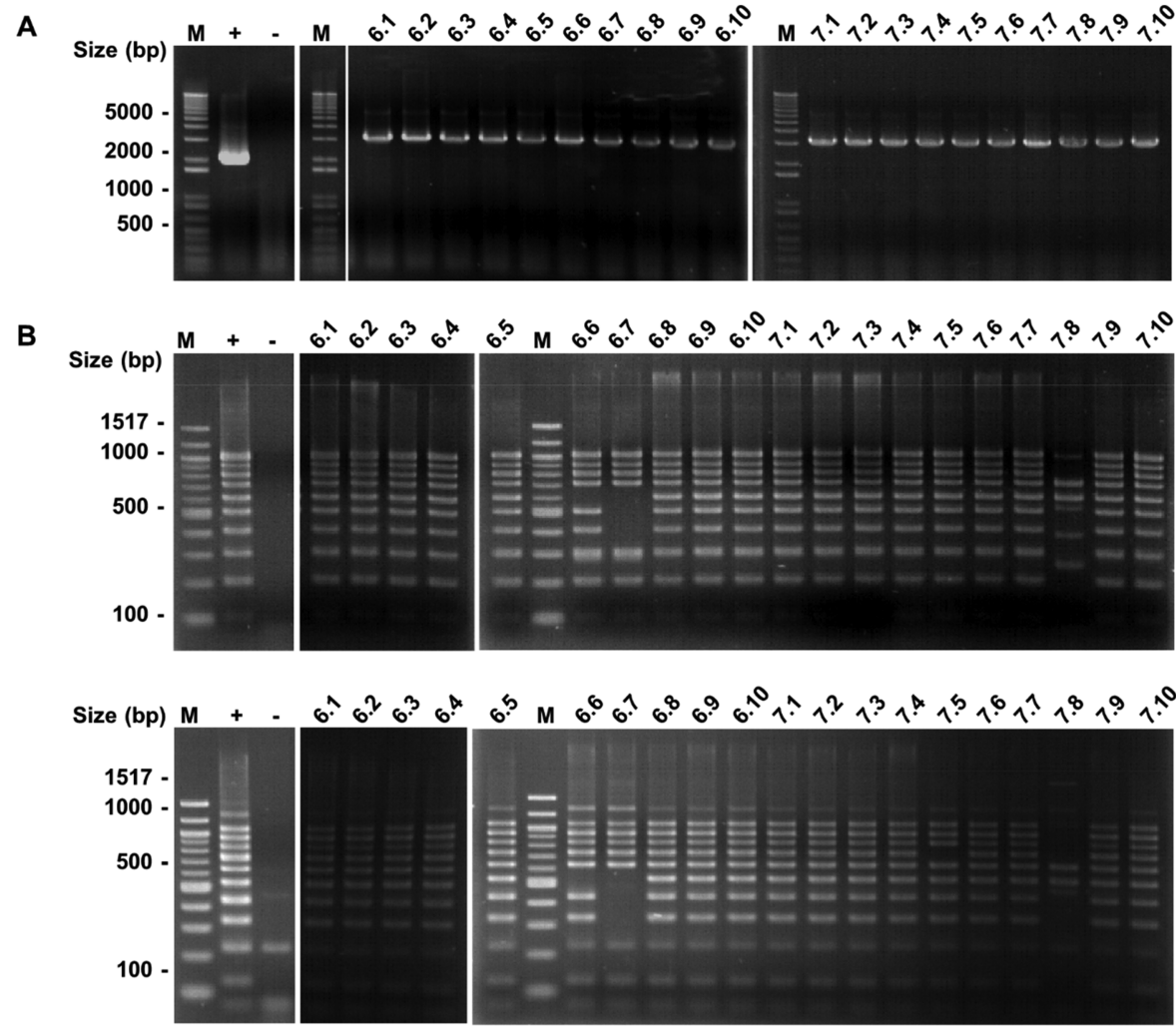

C

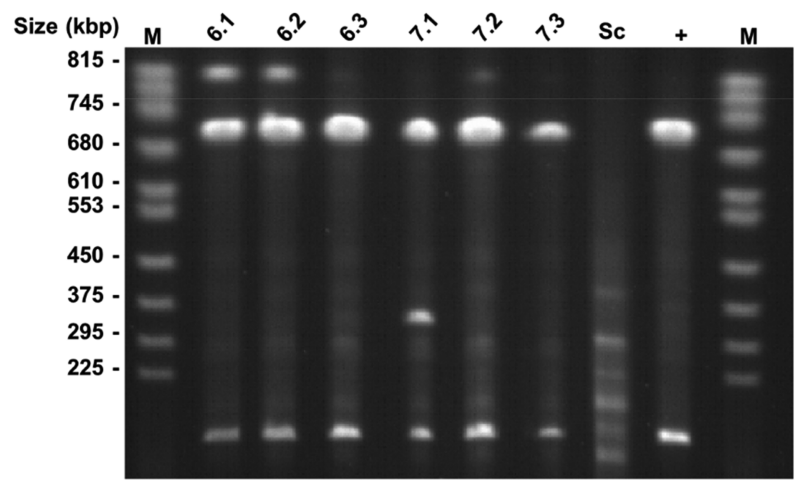

Figure 2. Screening of yeast transformants generated by CReasPy-cloning of M. pneumoniae M129 genome and deletion of MPN372. (A) The presence of the M. pneumoniae genome in the yeast and the desired target replacement were checked by simplex PCR analysis. Using M. pneumoniae specific primers flanking the target gene, a $3595 \mathrm{bp}$ amplicon was expected in properly edited clones, whereas a fragment of $2020 \mathrm{bp}$ was expected for wild types clones. (B) The completeness of the M. pneumoniae genome cloned in yeast was assessed by multiplex PCR using two sets of primers (Top: set 1, Bottom: set 2). Each set consisted in ten pairs of primers evenly distributed around the genome of M. pneumoniae, allowing the simultaneous amplification of ten fragments ranging from 100 to $1000 \mathrm{bp}$ (set 1) and from 125 to $1025 \mathrm{bp}$ (set 2) in $100 \mathrm{bp}$ increments. Clones carrying M. pneumoniae genomes without major rearrangement displayed a ten bands profile identical to the one obtained in the positive control. "M": DNA Ladder; “+”: M. pneumoniae M129 gDNA; “-": negative control without DNA. (C) The size of the M. pneumoniae genome cloned in yeast was assessed by enzymatic restriction and Pulsed Field Gel Electrophoresis (PFGE). Digestion of the bacterial genome with the restriction enzyme NotI should generate two linear DNA fragments of $711 \mathrm{kbp}$ and $105 \mathrm{kbp}$. "M": PFGE DNA ladder; "Sc": S. cerevisiae VL6-48N; “+”: M. pneumoniae M129. The images used to produce panels $\mathrm{A}-\mathrm{C}$ are spliced from multiple gels in order to display the same 20 yeast clones. Original images are available in Supplementary Figure S3.

simultaneously perform the cloning and editing of a bacterial genome.

To demonstrate the effectiveness of the CReasPy-cloning strategy, we cloned with a high efficiency the genome of Mycoplasma pneumoniae M129 23 in yeast, while simultaneously deleting a gene encoding for a virulence factor. Analysis showed that the cloned genome was intact and essentially error-free. We successfully applied the same strategy to Mycoplasma leachii strain PG50, ${ }^{24}$ in order to demonstrate that genomes cloned using CReasPy-cloning are suitable for genome transplantation.
We then stretched the capability of CReasPy-cloning by successfully editing two or three distinct loci simultaneously.

\section{RESULTS AND DISCUSSION}

Simultaneous Cloning and Engineering of Mycoplasma pneumoniae Genome in Yeast. We have developed a method dubbed "CReasPy-cloning", in order to perform the simultaneous cloning and engineering of megabase-sized genomes in yeast. Figure 1A outlines the general principle of the method. First, the yeast Saccharomyces cerevisiae strain VL6- 
$48 \mathrm{~N}$ is transformed with the plasmids pCas9 (TRP1) and pgRNA (URA3), allowing respectively the expression of a codon-optimized version of the Cas9 nuclease from Streptococcus pyogenes ${ }^{12}$ and a chimeric guide RNA (gRNA) merging the CRISPR RNA with the trans-activating crRNA. ${ }^{12,13,25,26}$ Then, the yeasts are cotransformed with the purified bacterial chromosome and a linear DNA cassette comprised of the yeast elements CEN and HIS, and ARS if needed ${ }^{7}$ (CEN: centromere; HIS3: histidine auxotrophic marker; ARS: autonomously replicating sequence). The cassette also bears an antibiotic resistance marker (used for an eventual back transplantation experiment later on ${ }^{27}$ ) and is flanked with recombination arms corresponding to the locus to edit (Figure S1). The Cas9/gRNA complex generates a double strand break at the targeted locus on the bacterial chromosome, which is then repaired by the homologous recombination machinery of the yeast using the provided template cassette. As a result, the target locus is edited, and the bacterial genome now bears the yeast elements cassette (CEN and HIS, and ARS if used) and is carried by the yeast as a large centromeric plasmid. After the CReasPy-cloning protocol, the yeast transformants selected from SD-His solid media plates, are screened using a combination of PCR analysis and chromosome size determination by PFGE, in order to check the integrity of the cloned genome.

As a proof of concept, we have used CReasPy-cloning to simultaneously clone and engineer the genome of the human pathogen M. pneumoniae M129 (816 kb, 40\% G + C), for which no efficient genome engineering tool is available. The addition of ARS element(s) is generally required for genomes having a $\mathrm{G}+$ $\mathrm{C} \%$ above $40 .{ }^{19-2}$ Since the $\mathrm{G}+\mathrm{C} \%$ of the M. pneumoniae M129 genome stands at the limit, we elected to work with ARSless and ARS containing linear DNA cassettes. Three genes encoding virulence factors were targeted: MPN372 encoding the CARDS toxin, ${ }^{28}$ MPN142 encoding a cytadherence protein, ${ }^{29,30}$ and MPN400 encoding an immunoglobulinbinding protein $^{31}$ (Figure $1 \mathrm{~B}$ and 1C). In the case of MPN400, we targeted either the single gene or the operon it belongs to (MPN398-400). In the case of MPN142, we targeted either the single gene or part of the operon it belongs to (MPN142-143), as the other genes in the operon (MPN140141) are suspected to be essential. ${ }^{32}$

Initially, a set of experiments targeting MPN372 was performed during which all the components (pgRNA, pCas9, chromosome and cassette) were cotransformed simultaneously in the yeast (Table S1). However, these experiments failed to yield positive clones. Therefore, an alternative protocol was set up, in which the pgRNA and pCas9 were transformed first, in order to give the yeast cells enough time to express the Cas9 nuclease and its partner gRNA before transformation with the bacterial chromosome. This strategy was much more efficient, and was used for the rest of the experiments described below. First, the CReasPy-cloning method was used to clone the M. pneumoniae genome and delete the gene MPN372 using an ARS-less recombination template (Figure 2). The presence of the M. pneumoniae genome cloned in yeast and the replacement of the target gene by the repair cassette were detected by simplex PCR. Twenty yeast transformants were screened, and all of them presented the expected profile with a single amplicon at $3595 \mathrm{bp}$ (Figure 2A). In a second step, a multiplex PCR was performed using two sets of 10 primer pairs, targeting a total of 20 loci evenly spread on the chromosome of $M$. pneumoniae (Figure S2), to check the completeness of the cloned genome. A total of 16 clones out of the 20 tested presented the expected 10 bands profile with both sets of primers (Figure 2B). Four clones (6.6, $6.7,7.5$, and 7.8) lacked one or more amplicons in one or both multiplex PCR, indicating that either one or several large parts of the bacterial genome had been lost during the CReasPy-cloning process. The cause of these large deletions in genomes that harbor the repair cassette at the correct location, is currently unknown, but might be linked to the documented off-target activity of Cas $9 .^{33-35}$ Indeed, if the nuclease cleaved the DNA at undesired loci, the yeast would need to perform a homologous recombination between two similar sequences elsewhere in the genome in order to maintain it. This phenomenon could be bolstered by the presence of multiple repeated regions in the genome of $M$. pneumoniae. ${ }^{36-38}$ The final transformants screening was based on the analysis of large DNA fragments generated by enzymatic restriction of the edited chromosome followed by PFGE. Among the 16 yeast clones validated by multiplex PCR, six were randomly selected and checked (Figure $2 \mathrm{C}$ ). Five clones presented the expected profile, identical to that of the nonedited genome, with two fragments at $711 \mathrm{kbp}$ and $105 \mathrm{kbp}$, for a chromosome size of $816 \mathrm{kbp}$. The extra upper band observed at the top of the gel, for clones 6.1 and 6.2, corresponds to partially digested DNA, with the chromosome migrating at a size matching $M$. pneumoniae linearized genome $(816 \mathrm{~kb})$.

In order to demonstrate the robustness of the CReasPycloning editing method, the exact same process was applied for the targeted deletions listed in Table 1.

Table 1. Screening of Yeast Transformants Generated by CReasPy-Cloning of M. pneumoniae M129

\begin{tabular}{lccc} 
& \multicolumn{2}{c}{ positive clones/analyzed clones $^{a}$} \\
\cline { 2 - 4 } targeted locus & simplex PCR & multiplex PCR & PFGE \\
MPN372 & $20 / 20$ & $16 / 20$ & $5 / 6$ \\
MPN142 & $19 / 20$ & $15 / 19$ & $4 / 6$ \\
MPN142-143 & $16 / 20$ & $11 / 16$ & $4 / 6$ \\
MPN400 & $20 / 20$ & $10 / 20$ & $5 / 6$ \\
MPN398-400 & $20 / 20$ & $14 / 20$ & $5 / 6$
\end{tabular}

${ }^{a}$ Five independent CReasPy-cloning experiments were performed, each targeting a different locus. For each experiment, the number of yeast transformants analyzed by simplex PCR, multiplex PCR, and PFGE is reported, as well as the number of positive clones obtained.

For the deletions MPN400 and MPN398-400, 100\% of the 20 tested clones were found positive by simplex PCR. When we targeted the MPN142 and MPN142-143 regions, 95\% and 80\% of the screened clones had the expected simplex PCR profiles, respectively (Table 1). At the next step, 79\%, 69\%, 50\%, and $70 \%$ of the clones validated by simplex PCR were found positive in multiplex PCR, for the deletion of MPN142, MPN142-143, MPN400, and MPN398-400, respectively. Finally, 67\%, 67\%, $83 \%$ and $83 \%$ of the clones tested by PFGE were positive, for MPN142, MPN142-143, MPN400, and MPN398-400, respectively (Table 1 ).

Overall, the CReasPy-cloning method we developed exhibits a high efficiency, as only a small number of clones (around 20) have to be screened to identify those carrying the expected edited bacterial chromosome. Using a yeast elements cassette which does not contain an $\mathrm{ARS}^{39}$ was identified as a key factor in reaching this high efficiency. The ARS acts as an origin of replication for the artificial yeast chromosome ${ }^{7}$ and must contain a copy of the essential 11 bp ARS Consensus Sequence (ACS): 5'-WTTTAYRTTTW-3'. It has been reported that 
removing the ARS element from TAR vector increased the efficiency of transformation. ${ }^{40}$ This improvement was linked to the fact that the recombination template tends to circularize instead of integrating into the bacterial chromosome during yeast transformation. This circular element bearing the ARS is efficiently replicated in yeast, leading to false-positive clones. In our case, the removal of the ARS element did not jeopardize the replication of the $M$. pneumoniae chromosome by the yeast machinery, as the $11 \mathrm{bp}$ ACS is present 19 times in the bacterial genome. During our work, we performed CReasPy-cloning experiment with an ARS-containing or an ARS-less recombination template (Figure S1), and obtained better efficiencies with the latter (Table S2). Depending on the locus targeted, 10 to 16 clones out of 20 were positive in multiplex PCR using an ARSless recombination template (i.e., 50-80\%) compared to only 1 to 4 with an ARS-containing recombination template (i.e., 5$20 \%)$.

In addition, several other genome cloning experiments were also performed in the yeast $S$. cerevisiae strain W303a, as this strain and the strain VL6-48N were both used in previous studies focused on the cloning and editing of mycoplasma genomes in yeast. ${ }^{7,41,42}$

Overall, similar transformation efficiencies were obtained for both strains (data not shown). Strain VL6-48N was retained for the rest of the experiments.

Application of CReasPy-Cloning to Multiple Mycoplasma Species. Following the successful development of our method for cloning and editing M. pneumoniae genome, we applied it on two other mycoplasma species: Mycoplasma leachii strain PG50 and Mycoplasma mycoides subsp. mycoides strain Afadé $(\mathrm{Mmm})$. The aims of these experiments were to (i) validate the CReasPy-cloning method for larger chromosomes, (ii) reclone mycoplasma genomes for which the yeast elements were previously inserted randomly by transposon, ${ }^{42}$ and (iii) check the ability of a CReasPy-cloned genome to be efficiently transplanted.

We succeeded in cloning $M$. leachii genome $(\sim 1 \mathrm{Mb})$ while inactivating MSB_0138, a gene encoding a beta-lactamase. We also successfully performed the cloning of $\mathrm{Mmm}$ genome $(\sim 1.2$ $\mathrm{Mb}$ ), while deleting of TS60_0301-0299, a trio of genes encoding an $\alpha$-glycerolphosphate oxidase, a glycerol kinase and a glycerol facilitator. Positive yeast clones carrying edited whole genomes were obtained for both species (Table 2 and Figure S4-S5). We observed that the cloning efficiencies of $\mathrm{Mmm}$ and $M$. leachii genomes (Table 2) were in the same range as those measured for M. pneumoniae, indicating that the CReasPycloning method is well suited for chromosomes in the megabase range.

Table 2. Screening of Yeast Transformants Generated by CReasPy-Cloning of M. mycoides subsp. mycoides and M. leachii

\begin{tabular}{ccccc} 
& & \multicolumn{3}{c}{ positive clones/analyzed } \\
clones $^{a}$ & \\
\cline { 3 - 5 } species & targeted locus & $\begin{array}{c}\text { simplex } \\
\text { PCR }\end{array}$ & $\begin{array}{c}\text { multiplex } \\
\text { PCR }\end{array}$ & PFGE \\
M. mycoides subsp. mycoides & TS60_0301- & $8 / 10$ & $4 / 8$ & $3 / 3$ \\
$\begin{array}{c}\text { strain Afadé } \\
\text { M. leachii strain PG 50 }\end{array}$ & MS99 & & & \\
MSB_0138 & $26 / 46$ & $9 / 14$ & $6 / 8$
\end{tabular}

${ }^{a}$ For each experiment, the number of yeast transformants analyzed by simplex PCR, multiplex PCR, and PFGE is reported, as well as the number of positive clones obtained.
In the case of $M$. leachii, the genome CReasPy-cloned in yeast of clones $9,34,36,37$, and 39 were isolated and transplanted in the recipient cell Mycoplasma capricolum, ${ }^{41}$ yielding between 10 and 50 colonies depending on the clone (cl. 8 yielded no transplant). Forty putative bacterial transplants were analyzed by simplex PCR (Figure S4): 36 were identified as edited $M$. leachii cells while the other 4 were identified as M. capricolum tetracycline spontaneous resistants. ${ }^{42}$ These experiments confirmed the robustness of the CReasPy-cloning as a fast and efficient method for the production of mutant strains of bacteria, provided that a transplantation protocol exists for these species. As such protocols are not currently available for M. pneumoniae and $\mathrm{Mmm}$, transplantation of their CReasPy-cloned genomes were not attempted during this study.

It is currently unknown whether the size of the target chromosome is a limiting factor for the "in-yeast cloning" process. Currently, the largest genomes carried in yeast in a single piece are the $1.8 \mathrm{Mbp}$ genome of Haemophilus influenzae ${ }^{17}$ and the $1.8 \mathrm{Mbp}$ genome of Spiroplasma citri. ${ }^{42}$ Interestingly, a recent experiment of chromosome fusion in $S$. cerevisiae has shown that the yeast can readily replicate a single chromosome of $11.8 \mathrm{Mbp}{ }^{43}$ This result suggests that the limit might not be the size of the chromosome to clone, but rather how much total DNA the yeast can carry $(6.8 \%$ excess for M. pneumoniae, $15 \%$ excess for $H$. influenzae). The advent of synthetic strains of $S$. cerevisiae could offer some insights, as these cells have significantly reduced genomes (around 1.1 Mbp removed from Sc2.0) ${ }^{44}$ and might allow to clone larger chromosomes.

Simultaneous Cloning and Multitarget Editing of Mycoplasma pneumoniae Genome. In order to improve our ability to efficiently create highly edited bacterial genomes, we evaluated if CReasPy-cloning could be used to perform simultaneous multitarget editings. To do so, we performed the deletion of either two or three loci: MPN372*/MPN400; MPN142-143*/MPN398-400; MPN372*/MPN142-143; MPN372*/MPN142/MPN400; MPN372*/MPN142-143/ MPN398-400. For each experiment, one locus was replaced by the yeast element cassette (indicated by a “*” next to the locus name) as the other locus or loci was simply deleted by homologous recombination with a DNA template. The multitarget protocol is based on the same principle as the single-target CReasPy-cloning, with few modifications (Figure 3).

First, the yeasts are "primed" by transformation with pCas9 and a multiguide pgRNA. To reduce the possibility of off-target cleavage by the Cas 9 nuclease, we elected to use the mutant eSpCas9 which was shown to have a higher specificity ${ }^{45}$ than the wild-type nuclease. Afterward, the cells are cotransformed with M. pneumoniae chromosome and 2 or 3 recombination templates. One of the template contains the CEN-HIS yeast elements, while the other or other two are clean deletion cassettes comprised of two juxtaposed 500 bp regions identical to the loci surrounding the gene to delete. These cassettes are tedious to produce (here by overlap PCR), but the large size of their recombination arms have a positive impact on the recombination efficiency, ${ }^{40}$ which offset the lack of a selection marker. Alternatively, $2 \times 45$ bp deletion cassettes, easily produced by annealing of two $90 \mathrm{bp}$ oligonucleotides, can be used and have been shown to be efficient to engineer the genome of S. cerevisiae ${ }^{12}$ or to edit a mycoplasma genome cloned in S. cerevisiae. ${ }^{13}$ After the multitarget CReasPy-cloning, yeast 

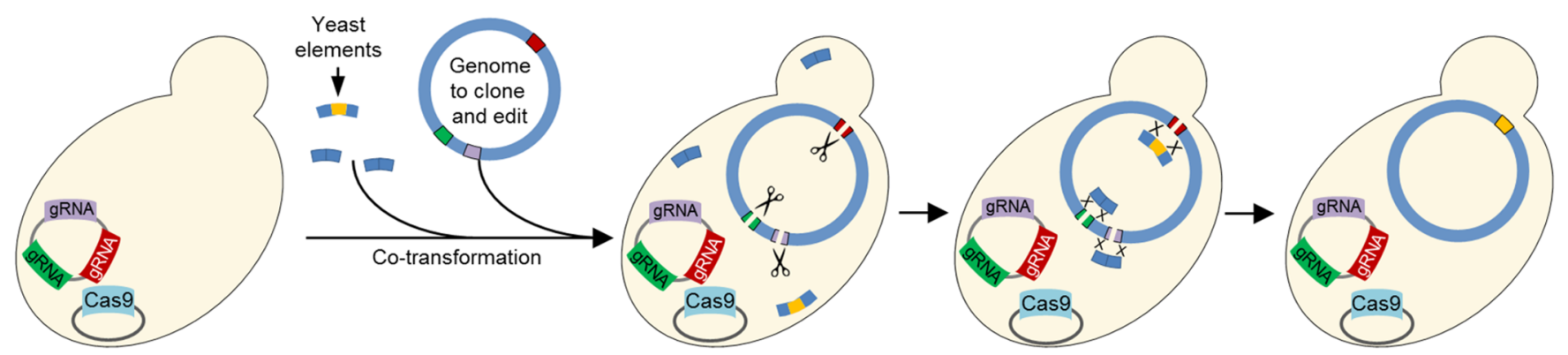

Figure 3. Overview of the multitarget CReasPy-cloning method. Yeast cells are transformed beforehand with two plasmids, allowing the expression of the Cas9 nuclease and multiple gRNA. The yeast is then transformed simultaneously with the target genome and three linear DNA fragments. One fragment contains the yeast elements flanked by two regions homologous to the target locus. The two remaining fragments are each comprised of two regions homologous to the target locus concatenated. Upon entry in the cell, the heterologous genome is cleaved at multiple loci by the Cas9/gRNA complexes, and subsequently repaired by the yeast homologous recombination system using the provided linear DNA templates. As a result, the heterologous chromosome now bears the yeast elements, inserted at a precise locus, and is now carried by the yeast as an artificial chromosome. In addition, the two other loci are edited, according to the experimenters design.

transformants were screened using the same process as in the single-target protocol.

Figure 4 illustrates an example of screening for the triple deletion MPN372*/MPN142/MPN400. Twenty yeast clones were first checked by simplex PCR for the deletion of MPN372 and its replacement by the yeast elements cassette (Figure 4A), which yielded 14 positive clones with the expected amplicon at $3596 \mathrm{bp}$. These positive clones were subsequently screened by PCR for the deletion of MPN142 (Figure 4B), yielding four positive clones presenting the expected $965 \mathrm{bp}$ band.

Finally, the deletion of MPN400 was assessed (Figure 4C), resulting in three positive clones presenting the expected 2156 bp band. The three clones obtained for the triple deletion were successfully validated by multiplex PCR (Figure 4D) and PFGE (Figure 4E).

All the multitarget deletions tested yielded positive clones (Table 3). As expected, due to the large number of cotransformed DNA fragments, the efficiency of the multitarget approach is significantly lower than that of the single-target CReasPy-cloning. Nonetheless, this efficiency is still high enough (minimum of $5 \%$ of the screened clones are fully validated) to warrant the screening of a relatively small set of transformants (i.e., < 50). It is interesting to note that the majority of the negative simplex PCR showed no amplification instead of amplification of the wild-type locus, suggesting that the targeted region is absent of the final chromosome. This high rate of unwanted deletions could be caused by the stochastic nature of the transformation process. Indeed, for the CReasPycloning process to occur properly, each cell must receive at least one copy of each recombination template. As there is a low probability of this perfect-case scenario to occur, many cells will lack one or more templates. As a result, the transformed chromosome will be cleaved at the desired site, but in the absence of a template the recombination will happen in a spurious manner between similar loci. This suggests that, although possible in theory, stretching the CReasPy-cloning strategy to four or five targets might not be practical. Nonetheless, the ability to simultaneously clone a megabasesized genome and edit three individual loci is still a significant improvement over existing strategies.

Whole Genome Sequencing of Genomes Cloned in Yeast by CReasPy-Cloning. Whole genome sequencing was performed on one of the genomes cloned and edited using CReasPy-cloning, in order to check whether our protocol had any mutagenic effects. We selected one clone from the double target experiment (MPN372*/MPN142-143 clone 4.8). Genomic DNA (including the cloned bacterial chromosome) was extracted from the yeast transformants and sequenced using both short reads (Illumina) and long reads technologies (Oxford Nanopore). As a control, the original M. pneumoniae M129 genome used for CReasPy-cloning experiments was resequenced using Illumina technology. De novo assembly of the genome of clone 4.8 was achieved using long and short reads, yielding a single contig. Global alignment of the clone 4.8 assembly with the expected genome design confirmed that no large deletion or chromosomal shuffling had occurred. In order to identify potential SNP or short indels, Illumina short reads from clone 4.8 and control M. pneumoniae M129 were mapped on the published genome sequence of $M$. pneumoniae strain M129 (Genbank ID NC_000912.1). The control clone of M. pneumoniae M129 used in our study presented 105 SNP and 19 indels compared to the M129 published genome. Those mutations were also identified in the genome of clone 4.8, suggesting that they occurred before the CReasPy-cloning process. Two additional mutations that were found only in clone 4.8. The first is an AAG to AAU transversion at position 182216 of the genome, leading to a $\mathrm{K}$ to $\mathrm{N}$ amino acid change in MPN141 (adhesin P1). The second is an insertion of ATGTTTG at position 452508 of the genome, in a region predicted to encode the ncRNA MPNnc041. The extent of the mutations observed in clone 4.8 suggest that the CReasPycloning method has no large mutagenic effect. However, SNP were expected as a result of natural genetic drift during the passages of either the mycoplasma or yeast cells in the laboratory.

In Vitro CReasPy-Cloning. In order to improve the flexibility of the CReasPy-cloning process, we attempted to perform the chromosome cleavage step in vitro (Figure S7). Indeed, this alternative strategy could offer several benefits compared to the in vivo method described above: (i) it would not be necessary to "prime" the yeast with the pgRNA and pCas9 plasmids, reducing the number of transformation steps to one, (ii) the selection markers used to maintain the pgRNA and pCas9 plasmids could be allocated for other purposes, (iii) a large number of different gRNA could be used simultaneously without the need to build a complex multi-gRNA plasmid, and (iv) the host cell genome could not be damaged by off-target activity, as the nuclease is not present in the cell. To do so, agarose plugs containing M. pneumoniae chromosomes were incubated in a mix of recombinant Cas 9 from $S$. pyogenes and 
A

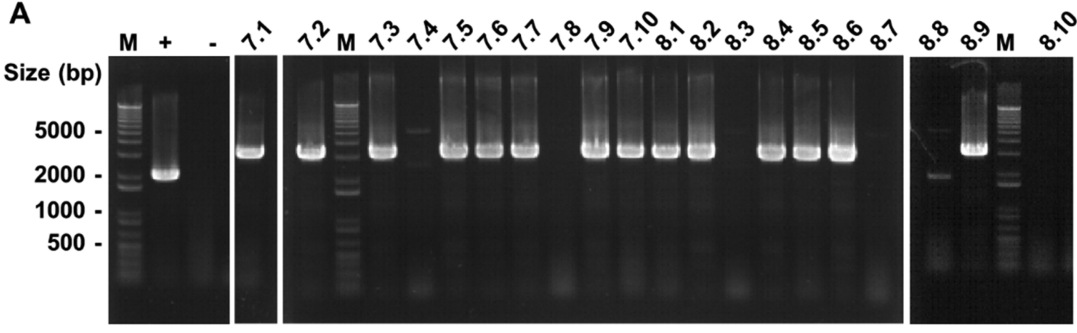

B

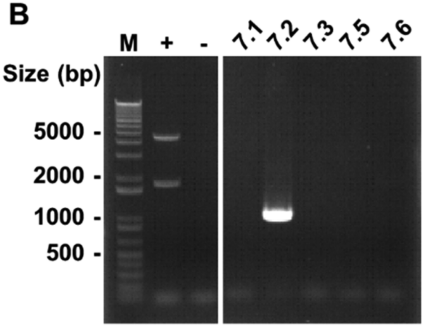

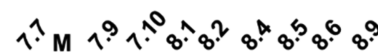
C
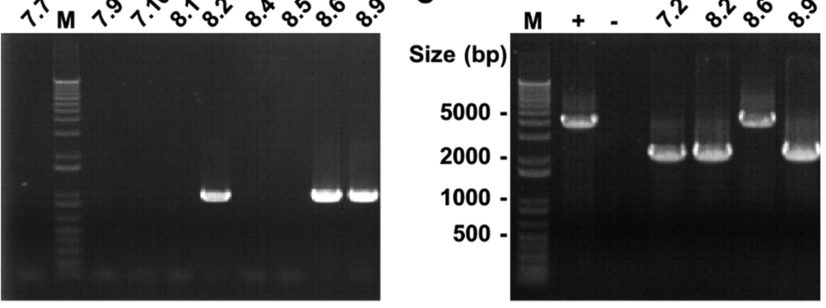

D Set 1

Set 2
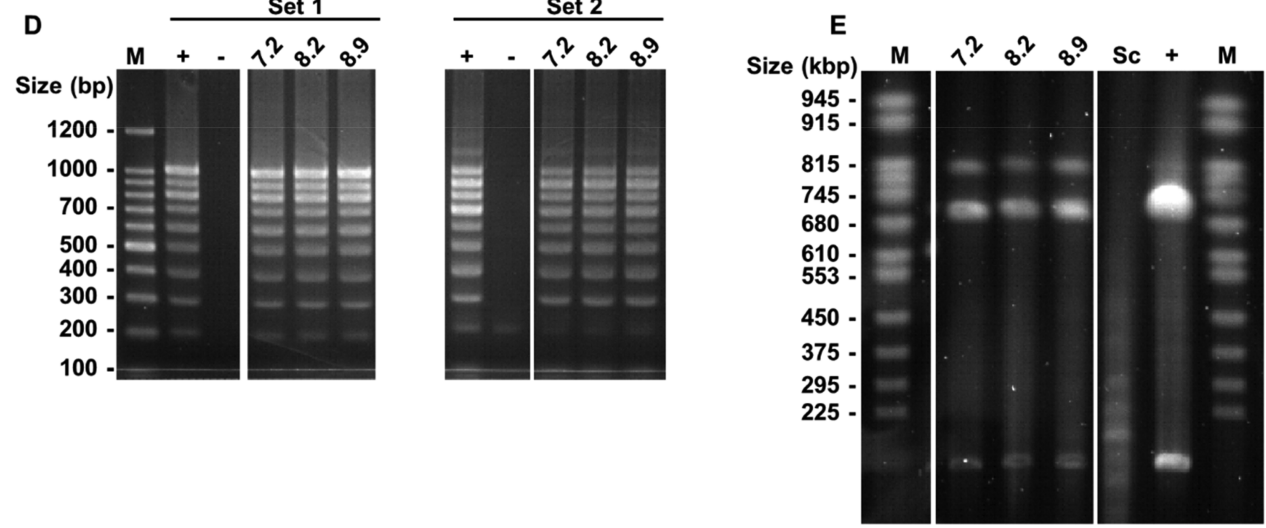

Figure 4. Screening of yeast transformants generated by CReasPy-cloning carrying Mycoplasma pneumoniae M129 genome and deletion of MPN372*/ MPN142/MPN400. (A) The presence of the M. pneumoniae genome in the yeast and the correct replacement of MPN372 were checked by simplex PCR analysis. Using $M$. pneumoniae specific primers flanking the target gene, an amplicon of $3595 \mathrm{bp}$ was expected in properly edited clones, whereas a fragment of $2020 \mathrm{bp}$ was expected for wild types clones. (B) The correct deletion of MPN142 was assessed by simplex PCR analysis. The expected amplicon size in clones bearing the deletion was $965 \mathrm{bp}$, whereas an amplicon of $4598 \mathrm{bp}$ was expected for clones bearing the wild-type genome. (C) The correct deletion of MPN400 was assessed by simplex PCR analysis. The expected amplicon size in clones bearing the deletion was 2156 bp, whereas an amplicon of $4048 \mathrm{bp}$ was expected for clones bearing the wild-type genome. (D) The completeness of M. pneumoniae genome in yeast was assessed by multiplex PCR using two sets of primers (see Figure 2 legend). Clones carrying M. pneumoniae genomes without major rearrangement displayed a ten bands profile identical to the one obtained in the positive control. "M": DNA Ladder; "+": M. pneumoniae M129 gDNA; "-": negative control without DNA. (E) The size of the M. pneumoniae genome in yeast was assessed by enzymatic restriction and pulsed-field gel electrophoresis (PFGE). Digestion by the restriction enzyme NotI-HF should yield two linear DNA fragments of $711 \mathrm{kbp}$ and $105 \mathrm{kbp}$. The extra upper band corresponds to M. pneumoniae genomes digested once producing a linear fragment of $816 \mathrm{~kb}$. "M": PFGE DNA ladder, "Sc": S. cerevisiae VL6-48N; “+”: M. pneumoniae M129. The images used to produce panels A-E are spliced from multiple gels in order to display the same 20 yeast clones. Original images are available in Supplementary Figure S6.

gRNA expressed by in vitro transcription from oligonucleotides. The Cas9-gRNA complex is able to diffuse through the agarose matrix and cleave the chromosome at the target site. The cut chromosome was subsequently cotransformed in yeast together with a recombination cassette bearing the yeast elements.

We validated this in vitro CReasPy-cloning strategy by targeting MPN400, using the same recombination cassette and gRNA spacer as those used in the in vivo approach. Ten yeast transformants were subsequently screened as described above (Figure S8): all the checked clones were positive by simplex PCR, and four were positive by multiplex. Of these, three were validated by PFGE. These results confirm the functionality of the in vitro approach, albeit with a slightly reduced efficiency compared to the in vivo strategy. Similar strategies, based on in vitro cleavage of DNA by CRISPR/Cas9 followed by capture in yeast have been highlighted by other groups, ${ }^{46,47}$ but these approaches are currently limited to sizes below $150 \mathrm{kbp}$ and are not suitable to work on whole bacterial genomes. To go on further, optimization of multiple parameters could be considered, including the concentration of Cas9 and gRNA, the incubation time and the amount of target chromosomes per agarose plug.

\section{CONCLUSION}

In this study, we developed the CReasPy-cloning tool, to efficiently clone megabase-sized DNA molecules in S. cerevisiae, while simultaneously editing up to three independent loci. This approach was originally conceived in order to improve the cloning process of bacterial genomes, in particular of the genus Mycoplasma, and to significantly shorten the time necessary to produce highly engineered strains for academic or applied purposes. $^{48}$ 
Table 3. Screening of Yeast Transformants Generated by Multitarget CReasPy-Cloning of M. pneumoniae M129

\begin{tabular}{cccccc} 
& \multicolumn{5}{c}{ positive clones/analyzed clones $^{a}$} \\
\cline { 2 - 6 } & $\begin{array}{c}\text { simplex } \\
\text { PCR yeast } \\
\text { elements }\end{array}$ & $\begin{array}{c}\text { simplex } \\
\text { PCR } \\
\text { second } \\
\text { target }\end{array}$ & $\begin{array}{c}\text { simplex } \\
\text { PCR } \\
\text { third } \\
\text { target }\end{array}$ & $\begin{array}{c}\text { multiplex } \\
\text { PCR }\end{array}$ & PFGE \\
$\begin{array}{c}\text { MPN372*/ } \\
\text { MPN400 }\end{array}$ & $7 / 19$ & $6 / 7$ & NA $^{b}$ & $3 / 6$ & $3 / 3$ \\
$\begin{array}{c}\text { MPN142-143*/ } \\
\text { MPN398-400 }\end{array}$ & $26 / 28$ & $4 / 26$ & NA & $4 / 4$ & $2 / 4$ \\
$\begin{array}{c}\text { MPN372*/ } \\
\text { MPN142-143 }\end{array}$ & $9 / 13$ & $1 / 9$ & NA & $1 / 1$ & $1 / 1$ \\
$\begin{array}{c}\text { MPN372*/ } \\
\text { MPN142/ }\end{array}$ & $14 / 20$ & $4 / 14$ & $3 / 4$ & $3 / 3$ & $3 / 3$ \\
$\begin{array}{c}\text { MPN400 } \\
\text { MPN372*/ }\end{array}$ & $6 / 19$ & $2 / 6$ & $2 / 2$ & $1 / 2$ & $1 / 1$ \\
$\begin{array}{l}\text { MPN142- } \\
\text { 143/ }\end{array}$ & & & & & \\
MPN398-400 & & & & &
\end{tabular}

${ }^{a}$ Two experiments were performed for the double-target strategy, and two for the triple-target strategy. For each experiment, the number of yeast transformants analyzed by simplex PCR, multiplex PCR, and PFGE is reported, as well as the number of positive clones obtained. ${ }^{b}$ NA: Not applicable.

However, our method is not limited to this type of application, as its reliance on the CRISPR/Cas9 system and homologous recombination makes it highly versatile and able to process DNA from different origins and of various sizes, as the entire genomes of some viruses, bacteria, chloroplasts, and mitochondria or parts of more complex genomes as those of eukaryotic cells.

In particular, we propose that CReasPy-cloning could be a valuable tool to capture intact chromosomes of uncultivable microorganisms from environmental samples (Figure S10). A target sequence could be selected based on previous or cooccurring metagenomics analysis, even if only small contigs are available. By producing an appropriate gRNA and recombination cassette, the chromosome of interest could in effect be "fished-out" by the yeast from the complex mixture of environmental DNA. Once cloned in yeast, these genomes could be easily sequenced and assembled in a single contig, which might not be possible using shotgun metagenome sequencing for rare organisms. ${ }^{49}$

\section{METHODS}

Yeast and Bacterial Strains, Culture Conditions, and Plasmids. Saccharomyces cerevisiae strain VL6-48N (MATa, his3- $\Delta 200$, trp1- $\Delta 1$, ura3-52, lys2, ade2-101, met14) is grown at $30{ }^{\circ} \mathrm{C}$ in YPDA medium (Clontech). Yeast transformants are selected by growth in Synthetic Defined (SD) medium depleted for one or several amino-acids: SD-Trp, SD-His or SD-Trp-Ura (Clontech).

Mycoplasma pneumoniae strain M129 (ATCC 29342), Mycoplasma leachii strain PG50 (M. leachii) and Mycoplasma mycoides subsp. mycoides strain Afadé $(\mathrm{Mmm})$ are grown at 37 ${ }^{\circ} \mathrm{C}$ in SP5 medium. ${ }^{42}$ Escherichia coli strains DH10B, strain NEB5- $\alpha$ or strain NEB10- $\beta$ used for plasmid cloning are grown at $37^{\circ} \mathrm{C}$ in lysogenic broth (LB) medium supplemented with $100 \mu \mathrm{g} \cdot \mathrm{mL}^{-1}$ of ampicillin.

The pCas9 plasmid used in this study corresponds to the p414-TEF 1p-Cas9-CYC1t plasmid developed by DiCarlo et al. ${ }^{12}$ It was acquired from the Addgene repository (reference: \#43802). This plasmid is a centromeric plasmid with a CEN6/ ARSH4 origin that contains the TRP1 selection marker.
All pgRNA plasmids used here derived from the p426SNR52p-gRNA.CAN1.Y-SUP4t plasmid developed by DiCarlo et al., ${ }^{12}$ and further modified by Tsarmpopoulos et al. ${ }^{13}$ Plasmid p426-SNR52p-gRNA is a high-copy $2 \mu$ plasmid carrying the URA3 auxotrophic marker for selection. It was acquired from the Addgene repository (reference: \#43803). Tsarmpopoulos et al. ${ }^{13}$ replaced the CAN1.Y 20 bp spacer by an AarI cloning spacer in order to create a plasmid in which specific spacers can be easily inserted. This plasmid was named p426-SNR52pgRNA.AarI.Y-SUP4t (or pgRNA.AarI).

Oligonucleotides. All the oligonucleotides used for this study are supplied by Eurogentec and are described in Table S3.

Construction of gRNA Plasmids for Simple Target Deletion. The gRNA targeting the loci MPN372, MPN142 and MPN400 in the M. pneumoniae genome, MSB_A0138 in the $M$. leachii genome and TS60_0301-0299 in the $\bar{M} m m$ genome, are designed using the SSC tool (http://crispr.dfci.harvard.edu/ SSC/2 with default parameters. The corresponding pgRNA plasmids are constructed following the protocol described in Tsarmpopoulos et al., 2015. ${ }^{13}$ Briefly, the plasmid p426SNR52p-gRNA.AarI.Y-SUP4t ("pgRNA.AarI") contains all the elements necessary for the expression of the gRNA in yeast. ${ }^{12,13}$ The spacer component of the gRNA can be swapped out by restriction of the plasmid using AarI, followed by ligation of annealed oligonucleotides pairs. The resulting plasmids are transformed in E. coli and sequence verified.

Construction of gRNA Plasmid for Triple Targets Deletion. The cassettes allowing the expression of the gRNAMPN142 and gRNAMPN400 are first amplified by PCR from the plasmids pgRNAMPN142 and pgRNAMPN400 respectively. These two cassettes are subsequently cloned in the plasmid pgRNAMPN372, using the Gibson Assembly Cloning Kit (NEB). The resulting plasmid pgRNA-triple-target is transformed in $E$. coli and sequence verified.

Construction of gRNA Plasmids for Double Targets Deletion. The cassettes allowing the expression of the gRNAMPN142 and the gRNAMPN400 are PCR amplified from the plasmid pgRNA-triple-target. The resulting fragments are cloned in the linearized pgRNAMPN372 (described above), using the Gibson Assembly Cloning Kit (NEB), producing the plasmids pgRNAMPN372-142 and pgRNAMPN372-400, respectively. The pgRNAMPN142-400 plasmid is constructed by removing the gRNAMPN372 from the pgRNA-triple-target plasmid using the Q5 Site-Directed Mutagenesis Kit (NEB).

Plasmid Transformation in Yeast. Yeast are transformed using the lithium acetate protocol optimized by Gietz et al. ${ }^{50}$ One $\mu \mathrm{g}$ of purified plasmid (pCas9 (TRP1) and/or pgRNA (URA3)) is used for each transformation, and transformants are selected for auxotrophy complementation (SD-Trp or SD-TrpUra).

Construction of Recombination Templates. Recombination templates containing the yeast elements are produced by PCR amplification of the ARS/CEN/HIS/PSPuro or CEN/ HIS/PSPuro loci from the plasmid pMT85-PRS-PSpuro (Figure S11), using the Advantage 2 Polymerase kit (Clontech). Complementary $60 \mathrm{bp}$-ends to the target sequence on M. pneumoniae genome are added to the extremities of the cassettes by using 5'-tailed PCR primers. Recombination templates comprised only of the regions flanking the loci to delete (without yeast element) are produced either by (a) annealing of two $90 \mathrm{bp}$ oligonucleotides, with $45 \mathrm{bp}$ corresponding to each side of the region to be knocked-out (initial denaturing step of $5 \mathrm{~min}$ at $95^{\circ} \mathrm{C}$ and controlled cooling 
to $16{ }^{\circ} \mathrm{C}$ with a ramp of $0.1{ }^{\circ} \mathrm{C} . \mathrm{s}^{-1}$ ), or (b) overlap PCR of two DNA fragments of $500 \mathrm{bp}$ amplified from the M. pneumoniae genome using the Q5 High-Fidelity DNA Polymerase, with each 500 bp PCR fragments corresponding to the DNA sequence surrounding the loci to be deleted.

Isolation of Mycoplasma Chromosomes in Agarose Plugs. Mycoplasma cells are grown in SP5 media, and harvested either by scrapping the culture flask bottom in HEPES-Sucrose buffer (HEPES $8 \mathrm{mM}$, sucrose $272 \mathrm{mM}, \mathrm{pH}$ 7.4) for M. pneumoniae or by centrifugation and resuspension in $\mathrm{T} / \mathrm{S}$ buffer (10 mM Tris pH 6.5, $500 \mathrm{mM}$ sucrose) for $M$. leachii and $\mathrm{Mmm}$, The cell suspension is then embedded in $1 \%$ low-melt agarose plugs and treated using the CHEF Mammalian Genomic DNA Plug Kit (Biorad), according to the manufacturer's protocol. $^{41,42}$ This preparation method yields agarose plugs that contain the isolated and intact mycoplasma chromosomes. The quality of the genomic DNA is checked by digesting trapped genomes with 50 units of restriction enzymes from NEB per half of agarose plug (NotI-HF for M. pneumonia, XhoI for M. leachii, and BssHII for $\mathrm{Mmm}$ ) followed by a pulsed-field gel electrophoresis. Prior to yeast transformation, mycoplasma genomes are released by digestion of the agarose matrix with three units per plug of $\beta$-Agarase I (NEB), and the DNA concentration is measured using an Epoch Microplate Spectrophotometer (BioTek).

Yeast Transformation with Mycoplasma Chromosomes and Recombination Templates. Yeast cells carrying the pCas 9 and pgRNA plasmids are transformed as described by Kouprina and Larionov (2008). ${ }^{22}$ For the simple target deletion experiments, $100 \mu \mathrm{L}$ of yeast spheroplasts are mixed with $2 \mu \mathrm{g}$ of genomic DNA and $300 \mathrm{ng}$ of recombination template containing the yeast elements. For the multiple target deletion experiments, $100 \mu \mathrm{L}$ of yeast spheroplasts are mixed with $2 \mu \mathrm{g}$ of genomic DNA, $300 \mathrm{ng}$ of recombination template containing the yeast elements and 0.5 or $1 \mu \mathrm{g}$ of each recombination template without the yeast elements. After transformation, the yeast cells are selected on SD-His solid agar plates containing 1 $\mathrm{M}$ of sorbitol, for 4 days at $30^{\circ} \mathrm{C}$. Individual colonies are picked and streaked on SD-His plates and incubated 2 days at $30^{\circ} \mathrm{C}$. Then, one isolated colony per streak is patched on the same medium and incubated for 2 days at $30{ }^{\circ} \mathrm{C}$.

Screening of Yeast Transformants Carrying Mycoplasma Genome. Total genomic DNA is extracted from yeast transformants according to Kouprina and Larionov (2008). ${ }^{22}$ Positive clones are screened for both the presence of the Mycoplasma genome and the correct deletion of the target gene by PCR, using the Advantage 2 Polymerase kit (Clontech) and specific primers located on either side of the target locus. Yeast transformants are then screened for bacterial genome completeness by multiplex PCR using two sets of PCR primers for M. pneumoniae (Table S3) and one set of primers for M. leachii and for Mmm (Table S3). Each set is comprised of ten pairs of primers evenly distributed across the bacterial genomes allowing the simultaneous amplification of ten fragments ranging from $\sim 100$ to $\sim 1000 \mathrm{bp}$, in $\sim 100 \mathrm{bp}$ increments. Clones carrying mycoplasma genomes with no major rearrangements display a characteristic ten bands ladder-profile with each primer set. The multiplex PCR are performed using the Qiagen Multiplex PCR Kit according to the manufacturer's instructions.

Yeast clones appearing positive by multiplex PCR are ultimately analyzed by restriction digestion and pulsed-field gel electrophoresis (PFGE) to assess the size of the mycoplasma chromosome. To do so, yeast cells are grow in SD-His media, harvested, embedded in agarose plugs and lysed by treatments with zymolyase, proteinase $\mathrm{K}$, and detergents to yield intact chromosomes. At this stage, yeast plugs carrying M. pneumoniae genomes are treated slightly differently compared to those containing M. leachii or Mmm genomes. For M. pneumoniae, the agarose-embedded yeast DNA is digested overnight with SgrDI (50 U/1/2 plug) from Thermo Scientific and submitted to a first PFGE (1\% agarose, $0.5 \times \mathrm{TBE}$ ) during $24 \mathrm{~h}$, with a switch time of $50-90 \mathrm{~s}$, at $6 \mathrm{~V} \mathrm{~cm}^{-1}$, an angle of $120^{\circ}$ and a temperature of 14 ${ }^{\circ} \mathrm{C}$. The plugs are then treated overnight with Plasmid-Safe ATP-dependent DNase (50 U/1/2 plug) from Epicenter and loaded on a standard gel ( $1 \%$ agarose, $1 \times$ TAE, $120 \mathrm{~min}$ at 120 $\mathrm{V})$. The plugs are finally digested overnight with NotI-HF (50 $\mathrm{U} / 1 / 2$ plug) and submitted to a second PFGE for $22 \mathrm{~h}$ (using the parameters previously described). The two first steps, both performed to electrophorese the linear yeast DNA out of the plugs while preserving the circular M. pneumoniae chromosome, turned out to be necessary to visualize the M. pneumoniae genome on gel at the end of the process. Yeast plugs carrying M. leachii or $\mathrm{Mmm}$ genomes are hydrolyzed with a cocktail of restriction enzymes (AsiSI, FseI and RsrII) and submitted to classical electrophoresis. Then, after the electroremoval of the yeast linear chromosomes, the DNA remaining in plugs is restricted with XhoI (M. leachii) and BssHII (Mmm) and submitted to PFGE. Pulse times are ramped from 60 to $120 \mathrm{~s}$ for $24 \mathrm{~h}$ at $6 \mathrm{~V} \mathrm{~cm}^{-1}$. Agarose gels are stained with SYBR Gold Nucleic Acid Gel Stain (Invitrogen) and PFGE patterns are scanned using the Vilbert Lourmat E-BOX VX2 Complete Imaging system.

Genome Sequencing. Genomic DNA of yeast MPN372*/ MPN142-143 clone 4.8 clone harboring edited M. pneumoniae chromosome was purified using Qiagen Genomic-Tips 100/G and Genomic DNA Buffers as described in Istace et al. (2017). ${ }^{51}$ DNA sequencing was performed at the Genome Transcriptome Facility of Bordeaux (https://pgtb.cgfb.u-bordeaux.fr) on a GridION (Oxford Nanopore, release 18.02, flowcell R9.4.A RevD) sequencer and a MiSeq sequencer (Illumina) using paired ends libraries. ONT sequencing generated $\sim 1870000$ reads whereas $\sim 3000000$ read pairs were obtained with Illumina technology. De novo assembly process included the following steps: (1) filtering long reads with Filtlong v0.2.0 (sequences $>1000$ bp score phred $\geq 9$, https://github.com/ rrwick/Filtlong), (2) selection of long reads mapping M. pneumoniae genome with Minimaps 2:v2.15-r905 $5^{52}$ (73676 reads selected), (3) assembly using Minimap/minimiasm ${ }^{53}$ (Miniasm 0.3-r179: https://github.com/lh3/miniasm), correction and polishing with Racon v1.3.1 ${ }^{54}$ ( 4 cycles) and Pilon ${ }^{55}$ v1.23 (4 cycles) tools. Illumina short reads used for polishing were trimmed with Trimmomatic ${ }^{56} 0.38$ (MINLEN:35, SLIDINGWINDOW:5:25). Whole genome alignment was performed with progressive MAUVE (version 20150226 and ref PMID:20593022). For short reads mapping onto M129 reference genome, data processing including quality check, trimming, alignment with BWA (Galaxy Version 1.2.3) and variant calling using Varscan (Galaxy Version 0.1) was completed using Galaxy instance (https://usegalaxy.org/). ${ }^{57}$

In Vitro CReasPy-Cloning. Agarose plugs containing M. pneumoniae chromosomes are incubated overnight at 37 ${ }^{\circ} \mathrm{C}$ in the presence of $1 \mu \mathrm{L}$ of Cas9 Nuclease from Streptococcus pyogenes (M0386T, NEB) and $21 \mu \mathrm{g}$ of gRNA transcripts produced by in vitro transcription of oligonucleotides (HiScribeTM T7 High Yield RNA Synthesis kit from NEB). Plugs are then treated overnight at $50{ }^{\circ} \mathrm{C}$ with $10 \mu \mathrm{L}$ of 
proteinase $\mathrm{K}$, washed several times in Tris $20 \mathrm{mM}$ pH8, EDTA $50 \mathrm{mM}$ and released from agarose gel by treatment with $\beta$ Agarase I ( 3 units/plug). Yeast cells are finally transformed as previously described: $100 \mu \mathrm{L}$ of yeast spheroplasts are mixed with $\sim 4 \mu \mathrm{g}$ of genomic DNA ( $\sim 50 \mu \mathrm{L}$ of gDNA plus $50 \mu \mathrm{L}$ of TE $1 \times)$ and $300 \mathrm{ng}$ of recombination template containing the yeast elements. The yeast transformants are selected and screened as described above.

\section{ASSOCIATED CONTENT}

\section{S Supporting Information}

The Supporting Information is available free of charge on the ACS Publications website at DOI: 10.1021/acssynbio.9b00224.

Figures S1-S11: A schematic view of the yeast elements recombination cassettes, a graphical map of M. pneumoniae M129 genome, the screening of VL6-48N yeast transformants carrying Mycoplasma leachii and Mycoplasma mycoides subsp. mycoides genomes, full images used to produce the panels in Figures S2, S4 and S8, the principle of the in vitro CReasPy-cloning, the screening of yeast transformants generated by in vitro CReasPy-cloning, a proposed application of the CReasPy-cloning to capture the genome of uncultivable micro-organisms and the map of the plasmid pMT85PSpuroM-PSlacZ-pRS313; Tables S1 and S2: An assessment of the number of genetic elements that can be cotransformed into yeast during CReasPy-cloning and a comparison of the CReasPycloning efficiency depending on the utilization of a yeast element cassette containing or not an ARS sequence (PDF)

Table S3: All the oligonucleotides used in this study (XLSX)

\section{AUTHOR INFORMATION}

\section{Corresponding Author}

*E-mail: carole.lartigue-prat@inra.fr. Tel: +33 557122359 .

Fax: +33557122369.

\section{ORCID 1}

\section{Carole Lartigue: 0000-0001-5550-7579}

\section{Author Contributions}

E.R., M.L-S., Y.A., and C.L. conceived and designed the research. E.R., V.T., M-P.D., G.G., and C.L. performed laboratory experiments and analyzed the in vitro data. P.S.-P. performed genome sequencing design and subsequent analyses. F.S. performed de novo assembly of mycoplasma genome. E.R., Y.A., P.S.P., and C.L. wrote the paper. All authors approved the final version of the paper.

\section{Notes}

The authors declare no competing financial interest.

The sequences from this study are available from the NCBI under SRA accession no. PRJNA563311.

\section{ACKNOWLEDGMENTS}

The authors thank all the members of the Genome Transcriptome Facility of Bordeaux for genome sequencing (https:// pgtb.cgfb.u-bordeaux.fr). They also thank Géraldine Gourgues, Dr. Fabien Labroussaa, and Angélique Alonso-Marrau for skilled technical assistance, Dr. Iason Tsarmpopoulos for providing biological material and advice, and Pr. Alain Blanchard for revising the manuscript. This work is part of the European MiniCell project "A model-driven approach to minimal cell engineering for medical therapy" selected by ANR, in the frame of the ERASynBio second Joint Call for Transnational Research Projects (No. ANR-15-SYNB-0001-04). It has also been supported by the National Science Foundation (Grant No. IOS-1110151) and the European Union's Horizon 2020 research and innovation program under Grant Agreement No. 634942.

\section{REFERENCES}

(1) Griffiths, A. J., Miller, J. H., Suzuki, D. T., and Lewontin, R. C. (2000) Introduction to Genetic Analysis, De Boeck.

(2) Nicholl, D. S. T. (2008) An Introduction to Genetic Engineering, Cambridge University Press, DOI: 10.1017/CBO9780511800986.

(3) Lacks, S. A. (2003) Rambling and scrambling in bacterial transformation-a historical and personal memoir. J. Bacteriol. 185, 1-6.

(4) Adli, M. (2018) The CRISPR tool kit for genome editing and beyond. Nat. Commun. 9, 1911.

(5) Joung, J. K., and Sander, J. D. (2013) TALENs: a widely applicable technology for targeted genome editing. Nat. Rev. Mol. Cell Biol. 14, $49-55$.

(6) Russell, J. J., et al. (2017) Non-model model organisms. BMC Biol. $15,55$.

(7) Benders, G. a, et al. (2010) Cloning whole bacterial genomes in yeast. Nucleic Acids Res. 38, 2558-69.

(8) Gibson, D. G., et al. (2010) Creation of a bacterial cell controlled by a chemically synthesized genome. Science $329,52-6$.

(9) Hutchison, C. A., et al. (2016) Design and synthesis of a minimal bacterial genome. Science (Washington, DC, U. S.) 351, aad6253.

(10) Noskov, V. N., Segall-Shapiro, T. H., and Chuang, R. Y. (2010) Tandem repeat coupled with endonuclease cleavage (TREC): A seamless modification tool for genome engineering in yeast. Nucleic Acids Res. 38, 2570-2576.

(11) Chandran, S., et al. (2014) TREC-IN: gene knock-in genetic tool for genomes cloned in yeast. BMC Genomics 15, 1180.

(12) DiCarlo, J. E., et al. (2013) Genome engineering in Saccharomyces cerevisiae using CRISPR-Cas systems. Nucleic Acids Res. 41, 4336-4343.

(13) Tsarmpopoulos, I., et al. (2016) In-Yeast Engineering of a Bacterial Genome Using CRISPR/Cas9. ACS Synth. Biol. 5, 104-9.

(14) Ketner, G., Spencer, F., Tugendreich, S., Connelly, C., and Hieter, P. (1994) Efficient manipulation of the human adenovirus genome as an infectious yeast artificial chromosome clone. Proc. Natl. Acad. Sci. U. S. A. 91, 6186-90.

(15) García-Ramírez, J. J., et al. (2001) Dominance of virus over host factors in cross-species activation of human cytomegalovirus early gene expression. J. Virol. 75, 26-35.

(16) Karas, B. J., Suzuki, Y., and Weyman, P. D. (2015) Strategies for cloning and manipulating natural and synthetic chromosomes. Chromosome Res. 23, 57-68.

(17) Karas, B. J., et al. (2013) Direct transfer of whole genomes from bacteria to yeast. Nat. Methods 10, 410-412.

(18) Karas, B. J., et al. (2013) Assembly of eukaryotic algal chromosomes in yeast. J. Biol. Eng. 7, 1-12.

(19) Noskov, V. N., et al. (2012) Assembly of large, high G+C bacterial DNA fragments in yeast. ACS Synth. Biol. 1 (7), 267-273.

(20) Venetz, J. E., et al. (2019) Chemical synthesis rewriting of a bacterial genome to achieve design flexibility and biological functionality. Proc. Natl. Acad. Sci. U. S. A. 116 (16), 8070-8079.

(21) Zhou, J., et al. (2016) CasHRA (Cas9-facilitated Homologous Recombination Assembly) method of constructing megabase-sized DNA. Nucleic Acids Res. 44 (14), No. e124.

(22) Kouprina, N., and Larionov, V. (2008) Selective isolation of genomic loci from complex genomes by transformation-associated recombination cloning in the yeast Saccharomyces cerevisiae. Nat. Protoc. 3, 371-377.

(23) Xiao, L., et al. (2015) Comparative genome analysis of Mycoplasma pneumoniae. BMC Genomics 16, 610. 
(24) Wise, K. S., et al. (2012) Complete genome sequences of Mycoplasma leachii strain PG50T and the pathogenic Mycoplasma mycoides subsp. mycoides small colony biotype strain Gladysdale. J. Bacteriol. 194, 4448-9.

(25) Mali, P., et al. (2013) RNA-Guided Human Genome Engineering via Cas9. Science (Washington, DC, U. S.) 339, 823-826.

(26) Firrao, G., et al. (2013) Genome wide sequence analysis grants unbiased definition of species boundaries in 'Candidatus Phytoplasma'. Syst. Appl. Microbiol. 36, 539-48.

(27) Lartigue, C., et al. (2007) Genome transplantation in bacteria: changing one species to another. Science 317, 632-8.

(28) Becker, A., et al. (2015) Structure of CARDS toxin, a unique ADP-ribosylating and vacuolating cytotoxin from Mycoplasma pneumoniae. Proc. Natl. Acad. Sci. U. S. A. 112, 5165-70.

(29) Hansen, E. J., Wilson, R. M., and Baseman, J. B. (1979) Twodimensional gel electrophoretic comparison of proteins from virulent and avirulent strains of Mycoplasma pneumoniae. Infect. Immun. 24, $468-475$.

(30) Lipman, R. P., Clyde, W. A., Denny, F. W., and Denny, F. W. (1969) Characteristics of virulent, attenuated, and avirulent Mycoplasma pneumoniae strains. J. Bacteriol. 100, 1037-1043.

(31) Grover, R. K., et al. (2014) A structurally distinct human mycoplasma protein that generically blocks antigen-antibody union. Science 343, 656-61.

(32) Glass, J. I., et al. (2006) Essential genes of a minimal bacterium. Proc. Natl. Acad. Sci. U. S. A. 103, 425-430.

(33) Schaefer, K. A., et al. (2017) Unexpected mutations after CRISPR-Cas9 editing in vivo. Nat. Methods 14, 547-548.

(34) Zhang, X.-H., Tee, L. Y., Wang, X.-G., Huang, Q.-S., and Yang, S.H. (2015) Off-target Effects in CRISPR/Cas9-mediated Genome Engineering. Mol. Ther.-Nucleic Acids 4, No. e264.

(35) Lin, Y., et al. (2014) CRISPR/Cas9 systems have off-target activity with insertions or deletions between target DNA and guide RNA sequences. Nucleic Acids Res. 42, 7473-85.

(36) Rocha, E. P. C., and Blanchard, A. (2002) Genomic repeats, genome plasticity and the dynamics of Mycoplasma evolution. Nucleic Acids Res. 30, 2031-42.

(37) Himmelreich, R., et al. (1996) Complete sequence analysis of the genome of the bacterium Mycoplasma pneumoniae. Nucleic Acids Res. $24,4420-49$.

(38) Himmelreich, R., et al. (1997) Comparative analysis of the genomes of the bacteria Mycoplasma pneumoniae and Mycoplasma genitalium. Nucleic Acids Res. 25, 701-12.

(39) Newlon, C. S., and Theis, J. F. (1993) The structure and function of yeast ARS elements. Curr. Opin. Genet. Dev. 3, 752-758.

(40) Noskov, V. N., et al. (2001) Defining the minimal length of sequence homology required for selective gene isolation by TAR cloning. Nucleic Acids Res. 29, E32.

(41) Lartigue, C., et al. (2009) Creating bacterial strains from genomes that have been cloned and engineered in yeast. Science 325, 1693-6.

(42) Labroussaa, F., et al. (2016) Impact of donor-recipient phylogenetic distance on bacterial genome transplantation. Nucleic Acids Res. 44, 8501-11.

(43) Shao, Y., et al. (2018) Creating a functional single-chromosome yeast. Nature 560, 331-335.

(44) Richardson, S. M., et al. (2017) Design of a synthetic yeast genome Downloaded from. Science (Washington, DC, U.S.) 355, 10401044.

(45) Slaymaker, I. M., et al. (2016) Rationally engineered Cas9 nucleases with improved specificity. Science (Washington, DC, U. S.) $351,84-88$.

(46) Lee, N. C. O., Larionov, V., and Kouprina, N. (2015) Highly efficient CRISPR/Cas9-mediated TAR cloning of genes and chromosomal loci from complex genomes in yeast. Nucleic Acids Res. 43, e55.

(47) Jiang, W., et al. (2015) Cas9-Assisted Targeting of CHromosome segments $\mathrm{CATCH}$ enables one-step targeted cloning of large gene clusters. Nat. Commun. 6, 1-8.
(48) Jores, J., et al. (2019) Removal of a subset of non-essential genes fully attenuates a highly virulent Mycoplasma strain. Front. Microbiol. 10,664 .

(49) Forster, S. C., et al. (2019) A human gut bacterial genome and culture collection for improved metagenomic analyses. Nat. Biotechnol. 37, $186-192$.

(50) Gietz, R. D., Schiestl, R. H., Willems, A. R., and Woods, R. A. (1995) Studies on the transformation of intact yeast cells by the LiAc/ SS-DNA/PEG procedure. Yeast 11, 355-360.

(51) Istace, B., et al. (2017) De novo assembly and population genomic survey of natural yeast isolates with the Oxford Nanopore MinION sequencer. GigaScience 6, 1-13.

(52) Li, H. (2018) Minimap2: pairwise alignment for nucleotide sequences. Bioinformatics 34, 3094-3100.

(53) Li, H. (2016) Minimap and miniasm: fast mapping and de novo assembly for noisy long sequences. Bioinformatics 32, 2103-10.

(54) Vaser, R., Sović, I., Nagarajan, N., and Šikić, M. (2017) Fast and accurate de novo genome assembly from long uncorrected reads. Genome Res. 27, 737-746.

(55) Walker, B. J., et al. (2014) Pilon: an integrated tool for comprehensive microbial variant detection and genome assembly improvement. PLoS One 9, No. e112963.

(56) Bolger, A. M., Lohse, M., and Usadel, B. (2014) Trimmomatic: a flexible trimmer for Illumina sequence data. Bioinformatics 30, 21142120.

(57) Afgan, E., et al. (2016) The Galaxy platform for accessible, reproducible and collaborative biomedical analyses: 2016 update. Nucleic Acids Res. 44, W3-W10. 